\title{
Fruit and vegetable consumption and risk of cholecystectomy: a prospective cohort study of women and men
}

\author{
Caroline Nordenvall ${ }^{1,2} \cdot$ Viktor Oskarsson $^{3} \cdot$ Alicja Wolk $^{3}$
}

Received: 14 December 2015 / Accepted: 11 August 2016 / Published online: 20 August 2016

(C) The Author(s) 2016. This article is published with open access at Springerlink.com

\begin{abstract}
Purpose Epidemiologic data on whether consumption of fruit and vegetables (FVs) decreases the risk of gallstone disease are sparse. Therefore, we examined the association between FV consumption and the 14-year risk of symptomatic gallstone disease (defined as occurrence of cholecystectomy) in a large group of middle-aged and elderly persons.

Methods Data from two population-based cohorts were used, which included 74,554 men and women (born 19141952). Participants filled in a food frequency questionnaire in the late fall of 1997 and were followed up for cholecystectomy between 1998 and 2011 via linkage to the Swedish Patient Register. Cox regression models were used to obtain hazard ratios (HRs).

Results During 939,715 person-years of follow-up, 2120 participants underwent a cholecystectomy (1120 women and 1000 men). An inverse association between FV consumption and risk of cholecystectomy was observed in age- and sex-adjusted analyses $\left(P_{\text {trend }}=.036\right)$ but not in confounder-adjusted analyses $\left(P_{\text {trend }}=.43\right)$. The multivariable-adjusted HR was 0.95 (95\% CI 0.83-1.08) for the
\end{abstract}

Electronic supplementary material The online version of this article (doi:10.1007/s00394-016-1298-6) contains supplementary material, which is available to authorized users.

Caroline Nordenvall

caroline.nordenvall@ki.se

1 Department of Molecular Medicine and Surgery, Karolinska Institutet, Stockholm 171 76, Sweden

2 Center for Digestive Diseases, Department of Coloproctology, Karolinska University Hospital, 17176 Stockholm, Sweden

3 Institute of Environmental Medicine, Karolinska Institutet, 17177 Stockholm, Sweden highest compared with the lowest sex-specific quartile of FV consumption. There was no evidence of interactions with age $(P=.25)$ or sex $(P=.72)$ in analyses pooled by sex. However, an age-by-FV consumption interaction was observed in separate analyses of women $(P=.010)$, with decreased HRs of cholecystectomy for ages up to 60 years. Conclusions This study supports an inverse association between FV consumption and risk cholecystectomy in women, although the association was restricted to women aged 48-60 years. In contrast, the study does not support an association in men.

Keywords Fruit consumption - Vegetable consumption . Gallbladder disease $\cdot$ Cohort study

\section{Introduction}

In order to improve health and longevity, a high consumption of fruit and vegetables (FVs) is recommended as part of a healthy diet [1]. While a variety of chronic diseases have been studied with regard to FV consumption up to a meta-analysis level, such as type 2 diabetes [2], cardiovascular disease [3], and cancer [4-6]; its association with gallstone disease has seldom been studied, particularly in large prospective cohort studies. In the only such study, which was performed in the Nurses' Health Study (NHS), it was observed that women who were high consumers of FVs had a decreased risk of cholecystectomy (used as a proxy for symptomatic gallstone disease) [7]. In other studies (i.e., in small cohort, case-control, and cross-sectional studies), both inverse [8,9] and null [10-14] associations have been observed between FV consumption and risk of gallstone disease (defined as the presence of gallstones on ultrasound and/or occurrence of cholecystectomy). 
To investigate whether the findings from the NHS were possible to reproduce in a cohort of European women, as well as to provide large prospective data on men for the first time, we examined the association between FV consumption and the 14-year risk of cholecystectomy in two prospective cohorts of middle-aged and elderly persons.

\section{Methods}

\section{Cohorts}

The Swedish Mammography Cohort is a population-based cohort of 66,651 women, aged 40-75 years at recruitment in 1987-1990. All women answered a baseline questionnaire with questions on diet and lifestyle characteristics (i.e., marital status, education, height, and weight) and 39,227 of them (then aged 48-83 years) answered a second questionnaire in 1997 with expanded questions on diet and lifestyle characteristics as well as with additional questions on medication use, supplement use, and medical history. The Cohort of Swedish Men is a population-based cohort of 48,850 men, aged 45-79 years at recruitment in 1997, who answered a questionnaire that was identical to the second questionnaire in the Swedish Mammography Cohort. Details on the cohorts can be viewed at www.ki.se/en/imm/ unit-of-nutritional-epidemiology.

This analysis was restricted to women and men who completed the questionnaire in 1997 (because the first female questionnaire did not contain questions on a number of important co-variables, such as cigarette smoking, physical activity, and use of postmenopausal hormones; $n=88,077)$. At baseline, we excluded those with incorrect personal identity numbers $(n=540)$, implausible energy intakes $( \pm 3$ standard deviations of the sex-specific logtransformed mean; $n=1090$ ), and histories of cancer or cholecystectomy ( $n=10,413$ ) as well as those with missing data on fruit consumption or vegetable consumption ( $n=981)$. In addition, we excluded participants who developed cancer in the duodenum, liver, gallbladder, biliary ducts, or pancreas during follow-up $(n=499)$, and thus, 74,554 participants were left for analyses. Ethical approval was granted by the Regional Ethical Board at Karolinska Institutet.

\section{Dietary variables}

Participants reported their usual FV consumption over the past year as part of a 96-item food frequency questionnaire. For each item, one of 8 frequency-of-consumption responses could be chosen ("never," "1-3 times/month," "1-2 times/week," "3-4 times/week," "5-6 times/week,"

"1 time/day," "2 times/day," and " $\geq 3$ times/day"). There were 13 questions on vegetables (carrot, beetroot, lettuce, cabbage, cauliflower, broccoli, tomato, pepper, spinach, peas, onion, garlic, and other vegetables), five on fruit (orange, apple, banana, berry, and other fruit), and one on orange juice. In a validation study, which was conducted in a subsample of 129 women from the Swedish Mammography Cohort using a similar food frequency questionnaire (including 60 items), the Spearman correlation coefficients between the food frequency questionnaire and four 1-week diet records (completed 3-4 months apart) ranged from 0.4 to 0.7 (unpublished data). Frequency responses were converted to average consumption (servings/day), and the variable for total FV consumption was created by aggregating the consumption of all individual items [including that on orange juice, because the exposure-outcome association was highly similar with and without that item (data not shown)]. We also created variables for consumption of total fruit (including orange juice), total vegetables, citrus fruits (orange and orange juice), green leafy vegetables (lettuce and spinach), cruciferous vegetables (cabbage, cauliflower, and broccoli), and items high in vitamin $\mathrm{C}$ (cabbage, broccoli, pepper, orange, orange juice, berry, and other fruit).

Data on dietary co-variables (i.e., foods, beverages, and nutrients) were acquired from the food frequency questionnaire. Nutrients were adjusted for energy intake (1700 kcal/ day in women and $2600 \mathrm{kcal} /$ day in men) using the residual method [15].

\section{Other variables}

The questionnaire contained questions on education, smoking status, physical activity, use of aspirin, height and weight, hyperlipidemia, and diabetes. Body mass index (BMI) was calculated as weight (kg) divided by height squared $\left(\mathrm{m}^{2}\right)$. Additional data on the presence of hyperlipidemia and diabetes were obtained from the Swedish Patient Register and the Swedish Diabetes Register. The female questionnaire also contained questions on parity and use of oral contraceptives and postmenopausal hormones.

\section{Follow-up for cholecystectomy}

We performed follow-up for cholecystectomy between January 1, 1998, and December 31, 2011, via linkage to the Swedish Patient Register (represented by codes JKA20 and JKA21 in the Swedish Classification of Operations and Major Procedures). Data on cholelithiasis and cholecystitis in relation to the surgical procedure were obtained from the same register (represented by codes K80 and K81 in the International Classification of Diseases-10). Data on cancer and death were acquired from the Swedish Cancer Registry and the Swedish Cause of Death Register, respectively. 
Table 1 Age-standardized baseline characteristics by sex-specific quartiles of fruit and vegetable consumption

\begin{tabular}{|c|c|c|c|c|c|c|c|c|}
\hline \multirow[t]{3}{*}{ Characteristics $^{\mathrm{a}}$} & \multicolumn{8}{|c|}{ Quartiles of consumption (servings/day) (median) } \\
\hline & \multicolumn{4}{|c|}{$\operatorname{Men}(n=42,516)$} & \multicolumn{4}{|c|}{ Women $(n=32,038)$} \\
\hline & $<2.4(1.7)$ & $2.4-3.5(3.0)$ & $3.6-5.1(4.3)$ & $>5.1(6.5)$ & $<3.3(2.4)$ & $3.3-4.7(4.0)$ & $4.8-6.6(5.6)$ & $>6.6(8.3)$ \\
\hline No. of participants & 10,631 & 10,650 & 10,613 & 10,622 & 8013 & 8008 & 8009 & 8008 \\
\hline Age (years) (mean) & 61.0 & 59.7 & 59.3 & 59.7 & 63.6 & 61.6 & 61.0 & 60.2 \\
\hline Education >12 years $(\%)$ & 9.2 & 14.4 & 18.0 & 23.2 & 12.4 & 19.0 & 23.5 & 27.1 \\
\hline Current smoker $(\%)$ & 33.7 & 24.4 & 21.4 & 18.3 & 27.7 & 19.8 & 17.0 & 14.0 \\
\hline BMI $\left(\mathrm{kg} / \mathrm{m}^{2}\right)$ (mean) & 26.0 & 25.8 & 25.6 & 25.6 & 25.0 & 24.9 & 24.8 & 24.8 \\
\hline $\begin{array}{l}\text { Physical activity }>40 \text { min of walking/ } \\
\text { day }(\%)\end{array}$ & 27.8 & 32.0 & 34.2 & 37.3 & 30.0 & 33.3 & 36.8 & 42.0 \\
\hline Use of aspirin (\%) & 35.7 & 38.2 & 38.0 & 38.0 & 50.2 & 51.9 & 51.4 & 50.8 \\
\hline History of diabetes (\%) & 10.4 & 9.7 & 8.5 & 9.9 & 4.3 & 4.2 & 4.1 & 4.7 \\
\hline History of hyperlipidemia (\%) & 16.6 & 16.5 & 17.0 & 16.2 & 8.6 & 8.2 & 8.9 & 9.1 \\
\hline Ever used oral contraceptives (\%) & - & - & - & - & 58.1 & 59.4 & 60.9 & 60.2 \\
\hline Parity (mean) & - & - & - & - & 2.1 & 2.1 & 2.1 & 2.2 \\
\hline Ever used HRT $(\%)^{\mathrm{b}}$ & - & - & - & - & 50.6 & 54.6 & 56.2 & 58.1 \\
\hline \multicolumn{9}{|l|}{ Daily intake (mean) } \\
\hline Alcohol $(\mathrm{g})^{\mathrm{c}}$ & 15.4 & 15.0 & 15.0 & 14.9 & 6.4 & 6.7 & 6.7 & 6.9 \\
\hline Coffee (cups) & 3.7 & 3.5 & 3.4 & 3.3 & 3.2 & 3.1 & 3.0 & 3.0 \\
\hline Energy (kcal) & 2390 & 2586 & 2714 & 2992 & 1513 & 1680 & 1790 & 2026 \\
\hline
\end{tabular}

$B M I$ body mass index, HRT hormone replacement therapy

${ }^{a}$ Means and percentages were calculated for men and women with complete data. The percentage of missingness was $0.3 \%$ for education, $1.5 \%$ for smoking status, $3.5 \%$ for BMI, $8.6 \%$ for physical activity, $9.9 \%$ for use of aspirin, $1.0 \%$ for use of oral contraceptives, $5.8 \%$ for use of HRT, $2.4 \%$ for alcohol intake, and $4.9 \%$ for coffee consumption

b Calculated for postmenopausal women

${ }^{c}$ Calculated for current drinkers

\section{Statistical analyses}

We used Cox regression models to obtain hazard ratios (HRs) according to sex-specific quartiles of FV consumption (with attained age during follow-up as time-axis) [16]. Entry time was defined as age at January 1, 1998, and exit time was defined as age at cholecystectomy, death, or December 31, 2011 (end of follow-up).

Potential confounders were selected on the basis of previous studies on the risk of cholecystectomy in the Swedish Mammography Cohort and the Cohort of Swedish Men $[17,18]$ and on the basis of other studies that have examined the association between $\mathrm{FV}$ consumption and risk of gallstone disease [7-14]. Included in the multivariable analyses were sex, education, smoking status, alcohol drinking, use of aspirin, energy intake, coffee consumption, and physical activity (measured as time spent walking; see Table 2, footnotes, for categorization). Adjusting for other aspects of physical activity (i.e., exercise, work occupation, and household work) had negligible influence on the results (data not shown). Further adjustment for (1) potential intermediates [i.e., BMI $\left(<25,25-29, \geq 30 \mathrm{~kg} / \mathrm{m}^{2}\right)$, hyperlipidemia (no, yes), and diabetes (no, yes)], (2) nutrient intakes [i.e., sex-specific quartiles (g/day) of total fat, dietary fiber, carbohydrates, and protein, with the aim to examine the association between FV consumption and risk of cholecystectomy independent of these nutrients], and (3) female-specific factors [i.e., parity (nulliparous, parous) and use of oral contraceptives (never, ever) and postmenopausal hormones (premenopausal, never, ever)] did not change the results. Missing data on co-variables (see Table 1, footnotes) were handled using the missing indicator approach. Sensitivity analyses were performed using the complete-case approach, without any clear change in the results (data not shown).

A test for proportionality of hazards according to quartiles of FV consumption was conducted with the Schoenfeld test [19]. In practice, this corresponds to a test for an interaction with age when attained age is the time-axis. In the case of formal evidence of non-proportionality, we allowed hazards to vary flexibly by including an interaction term between FV consumption (in quartiles) and attained age (as a continuous variable, modeled using 3-knot restricted cubic splines) [20]. We also tested for an 
Table 2 Hazard ratios of cholecystectomy by sex-specific quartiles of fruit and vegetable consumption

\begin{tabular}{lllll}
\hline & \multicolumn{2}{l}{ Quartile of consumption $^{\mathrm{a}}$} & \multicolumn{1}{c}{$P$ for trend $^{\mathrm{b}}$} \\
\cline { 2 - 5 } & 1 & 2 & 3 & 18,630 \\
\hline No. of participants & 18,644 & 18,658 & 18,622 & $519 / 238,624$ \\
No. of cases/person-years & $532 / 227,763$ & $534 / 235,426$ & $535 / 237,903$ & - \\
Hazard ratio (95 \% CI) & & & & $0.88(0.78-0.99)$ \\
Age- and sex-adjusted & 1.00 (ref) & $0.94(0.83-1.06)$ & $0.92(0.82-1.04)$ & 0.036 \\
Multivariable-adjusted $^{c}$ & 1.00 (ref) & $0.96(0.85-1.09)$ & $0.96(0.85-1.09)$ & $0.95(0.83-1.08)$ \\
\hline
\end{tabular}

a See Table 1 for range (servings/day) of sex-specific quartiles of fruit and vegetable consumption in men and women

b Test for trend was calculated by modeling the sex-specific quartiles of FV consumption as a continuous variable

${ }^{c}$ Derived from a Cox regression model that was adjusted for attained age during follow-up (time-axis), sex, education ( $\leq 12$, $>12$ years), smoking status (never, past, current), alcohol drinking [never, past, current in $<$ or $\geq$ the sex-specific median intake (g/day)], physical activity $(<20$, $20-40,>40 \mathrm{~min}$ of walking/day, corresponding to approximate tertiles), use of aspirin (no, yes), energy intake [sex-specific quartiles (kcal/day)], and coffee consumption ( $<2,2-3,4-5, \geq 6$ cups/day)

interaction with sex (using the likelihood ratio test) and performed separate analyses of men and women as sensitivity analyses. Analyses were two-sided at the $5 \%$ significance level and performed with Stata 12.1 (StataCorp, TX, USA).

\section{Results}

A median of 4.1 daily servings of FVs were consumed at baseline (4.8 servings in women and 3.6 servings in men) including 1.4 servings of fruit (1.7 servings in women and 1.2 servings in men) and 2.5 servings of vegetables (2.9 servings in women and 2.2 servings in men). High consumers of FVs were less likely to be current smokers and more likely to be well educated, physically active, and users of postmenopausal hormones (Table 1). (See Electronic Supplementary Table S1 for baseline characteristics separated by fruit consumption and vegetable consumption.) During a total follow-up of 939,715 person-years (1998-2011: mean of 12.6 years and 25th percentile of 14.0 years), 2120 participants underwent a cholecystectomy (1120 women and 1000 men), of whom 2007 had a prior or concomitant diagnosis of cholelithiasis or cholecystitis.

In age- and sex-adjusted analyses, FV consumption was inversely associated with risk of cholecystectomy (Table 2). However, after adjustment for potential confounders, particularly for education, physical activity, and energy intake, the inverse association was attenuated. The multivariableadjusted HR was 0.95 (95\% CI 0.83-1.08) for the highest compared with the lowest sex-specific quartile of FV consumption (Table 2). Further adjustment for potential intermediates, that is, BMI, hyperlipidemia, and diabetes (HR 0.95 ; $95 \%$ CI $0.83-1.08$ ), as well as for intakes of total fat, dietary fiber, carbohydrates, and protein (HR 0.94; $95 \%$ CI 0.82-1.09), did not change the results. Null associations were also observed when various lag times between diet assessment and start of follow-up were applied [3 years ( $n=72,262$ including 1549 cases) to 5 years $(n=70,196$ including 1200 cases), with the aim to reduce the possibility that FV consumption had been affected by latent symptoms of gallstones] (data not shown). Likewise, the risk of cholecystectomy was not associated with consumption of total fruit (HR 0.98; $95 \%$ CI 0.86-1.13), total vegetables (HR 1.00; $95 \%$ CI 0.87-1.14), citrus fruits (HR 0.95; $95 \%$ CI $0.83-1.09$ ), green leafy vegetables (HR 1.09; $95 \%$ CI 0.94-1.26), cruciferous vegetables (HR 0.94; $95 \% \mathrm{CI}$ $0.83-1.07$ ), or items high in vitamin C (HR $0.93 ; 95 \% \mathrm{CI}$ $0.80-1.07)$.

There was no evidence of interactions between FV consumption and age $(P \geq .25)$ or sex $(P=.72)$ in relation to risk of cholecystectomy, with multivariable-adjusted HRs of 0.92 (95\% CI 0.76-1.10) and 0.97 (95\% CI 0.81-1.16) in men and women, respectively, for the highest compared with the lowest quartile of FV consumption. Further adjustment for parity and use of oral contraceptives and postmenopausal hormones resulted in a HR of 0.95 (95\% CI $0.80-1.14)$ in women. However, in contrast to the analyses pooled by sex, there was evidence of an interaction with age in the women who were high consumers of FVs $(P=.010$ for quartile 4 and $P \geq .51$ for quartile 2 and 3 ). A high FV consumption was associated with decreased HRs of cholecystectomy for ages up to 60 years, with seemingly linear decreases in magnitude in that age range, whereas for ages of more than 60 years, there was no such association (Fig. 1). Fifty-two percentage of the women were aged 60 years or less at baseline $(n=16,575)$, who contributed to $26 \%$ of the total number of person-years of follow-up $(n=106,560)$ and $34 \%$ of the total number of cases of cholecystectomy $(n=382)$ before they turned 61 years. No evidence of an interaction with age was observed in men $(P \geq .23)$. 


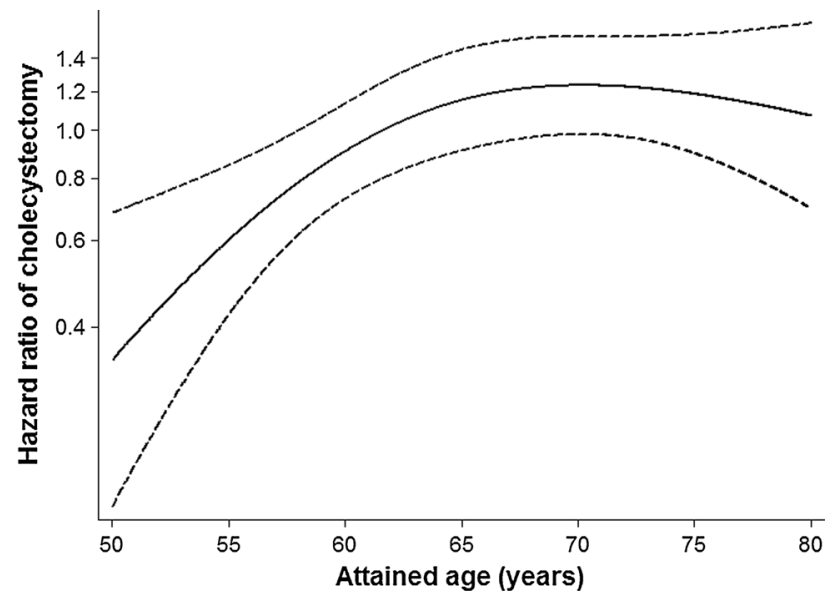

Fig. 1 Time-varying hazard ratios of cholecystectomy for the highest compared with the lowest quartile of fruit and vegetable consumption in women, according to attained age during follow-up (timeaxis). The figure illustrates time-varying hazard ratios and $95 \% \mathrm{CI}$ for the highest compared with the lowest quartile of fruit and vegetable consumption (Y-axis) according to attained age ( $X$-axis). Data were derived from a Cox regression model that included an interaction term between fruit and vegetable consumption (in quartiles) and attained age (as a continuous variable, modeled using 3-knot restricted cubic splines). Estimates were adjusted for the same covariables as those in Table 2

\section{Discussion}

In this large prospective cohort study, we found evidence of an inverse association between FV consumption and risk of cholecystectomy in women, although the association was restricted to women aged 48-60 years. In contrast, there was no evidence of an association in men.

Our finding of a null association with FV consumption in the combined analysis of women and men is in agreement with the few (and small) studies that have (1) examined its association with gallstone disease and (2) included both women and men [10-14], with the exception of a Spanish case-control study in which gallstone patients consumed less fruit than did control subjects [8]. However, on closer scrutiny, those results were solely driven by the female cases, as the male cases had a higher mean fruit consumption than had the control subjects. In fact, the only significant findings reported in the literature have been in women [7-9]. As such, our finding of an inverse association with FV consumption in women aged 60 years or less also finds some support. In a small case-control study of Greek women (mean age among control subjects 54 years), gallstone patients consumed less vegetables than did control subjects [9]. An inverse association with fruit consumption was in turn observed in the small case-control study of Spanish women mentioned above (mean age among control subjects 64 years) [8]. Finally, an inverse association with FV consumption was observed in the
NHS (mean age at the start of a 17-year follow-up period 50 years), the only large prospective cohort study to date [7]. It should be noted that most between-study comparisons are limited because of the mixture of study designs (i.e., cross-sectional, case-control, or cohort) and outcome definitions (i.e., presence of gallstones on ultrasound and/or occurrence of cholecystectomy).

Due to similarities in study design and outcome definition, a thorough comparison between the present study and the NHS is needed [7]. Among 77,090 women, followed up between 1984 and 2000, there was an inverse association between FV consumption and risk of cholecystectomy $\left(P_{\text {trend }}<.0001\right)$. The multivariable-adjusted HR was 0.79 (95\% CI 0.73-0.87) for the highest compared with the lowest quintile of FV consumption-and, in contrast to our study, there was no evidence of an interaction with age (HR for $<60$ years 0.80 and HR for $\geq 60$ years 0.78 ). Some differences between the two studies should also be noted, since they may affect generalizability and explain discrepancies in results. Firstly, and of importance regarding a potential interaction with age, the NHS had a younger age structure (aged 37-64 years at the start of a 17-year followup period) than had our cohort of women (aged 48-84 years at the start of a 14-year follow-up period). Secondly, adjustment for potential confounders had a minimal effect on the results in the NHS, which is a rather homogenous cohort of nurses, whereas the age- and sex-adjusted results in our population-based cohort were clearly attenuated in the multivariable-adjusted analyses. Finally, the crude incidence of cholecystectomy was very high in the NHS (6.2 cases per 1000 person-years), especially compared with that in our cohort of women (2.7 cases per 1000 person-years) as well as that in other European cohorts of women (2.8-3.4 cases per 1000 person-years) [21, 22].

Since gallstone disease is considerably more common in women than in men [23], which suggests sex differences in the etiology, it is plausible that the association with FV consumption might differ by sex. Higher levels of female sex hormones have been associated with an increased risk of gallstone disease, for example due to postmenopausal hormones [17, 21, 22, 24] (including in our data [17]) and multiple childbirths [25] [including in our data, with multivariable-adjusted HRs (95\% CI) of 1.37 (1.04-1.81), 1.43 (1.12-1.83), and 1.54 (1.20-1.98) for 1,2 , and $\geq 3$ childbirths, respectively, compared with nulliparity]. Experimental studies have demonstrated that overexpression of progesterone receptors may lead to a slow intestinal transit [26, 27], while clinical studies have shown that women's intestinal transits are slower than men's, which seem to depend on the menstrual cycle [28]. A slow intestinal transit may, in turn, increase the risk of gallstone disease by promoting bacterial overgrowth and by increasing the intestinal absorption of cholesterol [29-31]. FVs are rich in 
dietary fiber, which shortens the intestinal transit [32] and has been inversely associated with risk of gallstone disease [33]. Thus, it might be that an inverse association between FV consumption and risk of gallstone disease depends on female sex hormones, as well as the level of these, which may explain our finding of a lack of association in men and of an interaction with age in women.

Some strengths of our study were its large size, inclusion of women and men, prospective assessment of diet, and ability to control for a number of potential confounders. Other strengths included the population-based design and an almost complete follow-up via linkage to national health registers.

The major limitation of the study was the reliance on self-reported diet, which, inevitably, must have led to some degree of misclassification of FV consumption. Also, since the exposure was only measured once at baseline, further misclassification might have occurred as a result of potential changes in FV consumption during follow-up. However, due to the study's prospective design, such types of misclassification should have been unrelated to the future occurrence of cholecystectomy. Because of the age structure of the participants (aged 45-83 years at baseline), we could not determine how the risk of cholecystectomy is influenced by FV consumption earlier in life. Furthermore, we used gallstone disease that required cholecystectomy as the outcome of interest. Our results may, therefore, not be generalizable to patients with asymptomatic gallstone disease or to those with conservatively treated gallstone disease. However, apart from the fact that the probability of false-positive cases should have been low, we believe that patients treated with cholecystectomy represent the most clinically relevant fraction of gallstone disease. Since the cohorts consisted almost exclusively of non-Hispanic whites, the generalizability to populations of other ethnicities is also expected to be limited. On the other hand, the ethnically homogenous population should have kept the influence of confounding by genetic factors to a minimum. Finally, as in all observational studies, we cannot rule out the possibility of residual and/or unmeasured confounding (for example due to rapid weight loss and dieting [34], two co-variables for which we had no satisfactory data).

In conclusion, this prospective cohort study supports an inverse association between FV consumption and risk cholecystectomy in women, although the association was restricted to women aged 48-60 years. In contrast, the study does not support an association in men. Given the sparse epidemiologic data on the association between FV consumption and gallstone disease, particularly from large prospective cohort studies, further observational studies are required in both women and men.
Funding This work was supported by research grants from the Swedish Research Council/Committee for Infrastructure and the Board of Research at Karolinska Institutet (Distinguished Professor Award) (to Alicja Wolk). The funders had no role in the study design; the collection, analysis, or interpretation of the data; the writing of the manuscript; or the decision to submit the manuscript for publication.

\section{Compliance with ethical standards}

Conflict of interest The authors disclose no conflict of interest. All authors contributed to the study design and participated in the statistical analyses and the writing of the manuscript. All authors have read and approved the final version of the manuscript.

Ethics and consent Ethical approval was acquired from the Regional Ethical Board at Karolinska Institutet (Stockholm, Sweden), and return of a completed questionnaire was considered to imply informed consent.

Open Access This article is distributed under the terms of the Creative Commons Attribution 4.0 International License (http://creativecommons.org/licenses/by/4.0/), which permits unrestricted use, distribution, and reproduction in any medium, provided you give appropriate credit to the original author(s) and the source, provide a link to the Creative Commons license, and indicate if changes were made.

\section{References}

1. World Health Organization (2003) Diet, nutrition and the prevention of chronic diseases. Report of a Joint WHO/Food and Agriculture Organization Expert Consultation. World Health Organization Technical Report Series. Geneva

2. Carter P, Gray LJ, Troughton J, Khunti K, Davies MJ (2010) Fruit and vegetable intake and incidence of type 2 diabetes mellitus: systematic review and meta-analysis. BMJ 341:c4229. doi:10.1136/bmj.c4229

3. Hartley L, Igbinedion E, Holmes J, Flowers N, Thorogood M, Clarke A, Stranges S, Hooper L, Rees K (2013) Increased consumption of fruit and vegetables for the primary prevention of cardiovascular diseases. Cochrane Database Syst Rev 6:CD009874. doi:10.1002/14651858.CD009874.pub2

4. Aune D, Chan DS, Vieira AR, Rosenblatt DA, Vieira R, Greenwood DC, Norat T (2012) Fruits, vegetables and breast cancer risk: a systematic review and meta-analysis of prospective studies. Breast Cancer Res Treat 134(2):479-493. doi:10.1007/ s10549-012-2118-1

5. Aune D, Lau R, Chan DS, Vieira R, Greenwood DC, Kampman E, Norat T (2011) Nonlinear reduction in risk for colorectal cancer by fruit and vegetable intake based on meta-analysis of prospective studies. Gastroenterology 141(1):106-118. doi:10.1053/j.gastro.2011.04.013

6. Lunet N, Lacerda-Vieira A, Barros H (2005) Fruit and vegetables consumption and gastric cancer: a systematic review and metaanalysis of cohort studies. Nutr Cancer 53(1):1-10. doi:10.1207/ s15327914nc5301_1

7. Tsai CJ, Leitzmann MF, Willett WC, Giovannucci EL (2006) Fruit and vegetable consumption and risk of cholecystectomy in women. Am J Med 119(9):760-767. doi:10.1016/j. amjmed.2006.02.040

8. Ortega RM, Fernandez-Azuela M, Encinas-Sotillos A, Andres P, Lopez-Sobaler AM (1997) Differences in diet and food habits 
between patients with gallstones and controls. J Am Coll Nutr 16(1):88-95

9. Pastides H, Tzonou A, Trichopoulos D, Katsouyanni K, Trichopoulou A, Kefalogiannis N, Manousos O (1990) A case-control study of the relationship between smoking, diet, and gallbladder disease. Arch Intern Med 150(7):1409-1412

10. La Vecchia C, Decarli A, Pagano R (1998) Vegetable consumption and risk of chronic disease. Epidemiology 9(2):208-210

11. Misciagna G, Leoci C, Guerra V, Chiloiro M, Elba S, Petruzzi J, Mossa A, Noviello MR, Coviello A, Minutolo MC, Mangini V, Messa C, Cavallini A, De Michele G, Giorgio I (1996) Epidemiology of cholelithiasis in southern Italy. Part II: Risk factors. Eur J Gastroenterol Hepatol 8(6):585-593

12. Linos AD, Daras V, Linos DA, Kekis V, Tsoukas MM, Golematis V (1989) Dietary and other risk factors in the aetiology of cholelithiasis: a case control study. HPB Surg 1(3):221-227

13. Tseng M, DeVellis RF, Maurer KR, Khare M, Kohlmeier L, Everhart JE, Sandler RS (2000) Food intake patterns and gallbladder disease in Mexican Americans. Public Health Nutr 3(2):233-243

14. Volzke H, Baumeister SE, Alte D, Hoffmann W, Schwahn C, Simon P, John U, Lerch MM (2005) Independent risk factors for gallstone formation in a region with high cholelithiasis prevalence. Digestion 71(2):97-105. doi:10.1159/000084525

15. Willet W (1998) Nutritional epidemiology. Oxford University Press, New York

16. Cologne J, Hsu WL, Abbott RD, Ohishi W, Grant EJ, Fujiwara S, Cullings HM (2012) Proportional hazards regression in epidemiologic follow-up studies: an intuitive consideration of primary time scale. Epidemiology 23(4):565-573. doi:10.1097/ EDE.0b013e318253e418

17. Nordenvall C, Oskarsson V, Sadr-Azodi O, Orsini N, Wolk A (2014) Postmenopausal hormone replacement therapy and risk of cholecystectomy: a prospective cohort study. Scand J Gastroenterol 49(1):109-113. doi:10.3109/00365521.2013.858180

18. Nordenvall C, Oskarsson V, Wolk A (2015) Inverse association between coffee consumption and risk of cholecystectomy in women but not in men. Clin Gastroenterol Hepatol 13(6):10961102. doi:10.1016/j.cgh.2014.09.029

19. Schoenfled D (1980) Chi squared goodness-of-fit tests for proportional hazards regression model. Biometrika 67:145-153

20. Orsini N, Greenland S (2011) A procedure to tabulate and plot results after flexible modeling of quantitative covariate. Stata $\mathrm{J}$ 11:1-29

21. Liu B, Beral V, Balkwill A, Green J, Sweetland S, Reeves G, Million Women Study C (2008) Gallbladder disease and use of transdermal versus oral hormone replacement therapy in postmenopausal women: prospective cohort study. Bmj 337:a386. doi:10.1136/bmj.a386
22. Racine A, Bijon A, Fournier A, Mesrine S, Clavel-Chapelon F, Carbonnel F, Boutron-Ruault MC (2013) Menopausal hormone therapy and risk of cholecystectomy: a prospective study based on the French E3N cohort. CMAJ Can Med Assoc J 185(7):555561. doi:10.1503/cmaj.121490

23. Portincasa P, Moschetta A, Palasciano G (2006) Cholesterol gallstone disease. Lancet 368(9531):230-239. doi:10.1016/ S0140-6736(06)69044-2

24. Grodstein F, Colditz GA, Stampfer MJ (1994) Postmenopausal hormone use and cholecystectomy in a large prospective study. Obstet Gynecol 83(1):5-11

25. Liu B, Beral V, Balkwill A, Million Women Study C (2009) Childbearing, breastfeeding, other reproductive factors and the subsequent risk of hospitalization for gallbladder disease. Int $\mathbf{J}$ Epidemiol 38(1):312-318. doi:10.1093/ije/dyn174

26. Guarino M, Cheng L, Cicala M, Ripetti V, Biancani P, Behar J (2011) Progesterone receptors and serotonin levels in colon epithelial cells from females with slow transit constipation. Neurogastroenterol Motil 23(6):575-e210. doi:10.1111/j.1365-2982.2011.01705.x

27. Xiao ZL, Pricolo V, Biancani P, Behar J (2005) Role of progesterone signaling in the regulation of G-protein levels in female chronic constipation. Gastroenterology 128(3):667-675

28. Meier R, Beglinger C, Dederding JP, Meyer-Wyss B, Fumagalli M, Rowedder A, Turberg Y, Brignoli R (1995) Influence of age, gender, hormonal status and smoking habits on colonic transit time. Neurogastroenterol Motil 7(4):235-238

29. Heaton KW, Emmett PM, Symes CL, Braddon FE (1993) An explanation for gallstones in normal-weight women: slow intestinal transit. Lancet 341(8836):8-10

30. Kaur J, Rana SV, Gupta R, Gupta V, Sharma SK, Dhawan DK (2014) Prolonged orocecal transit time enhances serum bile acids through bacterial overgrowth, contributing factor to gallstone disease. J Clin Gastroenterol 48(4):365-369. doi:10.1097/ MCG.0b013e3182a14fba

31. Xie M, Kotecha VR, Andrade JD, Fox JG, Carey MC (2012) Augmented cholesterol absorption and sarcolemmal sterol enrichment slow small intestinal transit in mice, contributing to cholesterol cholelithogenesis. J Physiol 590(Pt 8):1811-1824. doi:10.1113/jphysiol.2011.224717

32. Muller-Lissner SA (1988) Effect of wheat bran on weight of stool and gastrointestinal transit time: a meta analysis. Br Med J 296(6622):615-617

33. Tsai CJ, Leitzmann MF, Willett WC, Giovannucci EL (2004) Long-term intake of dietary fiber and decreased risk of cholecystectomy in women. Am J Gastroenterol 99(7):1364-1370. doi:10.1111/j.1572-0241.2004.30153.x

34. Everhart JE (1993) Contributions of obesity and weight loss to gallstone disease. Ann Intern Med 119(10):1029-1035 